\title{
ICD-10. Postgraduate training course
}

MENTAL HEALTH AND THE ICD-10

\section{Norman Sartorius}

Division of Mental Health, World Health Organization, Geneva, Switzerland

A common language which can be understood by all who work in the field of mental health is a prerequisite for collaboration and consensus on key issues in mental health. To contribute to the development of such a common language the World Health Organization has initiated a number of projects, including the development of an internationally agreed and acceptable classification of mental disorders. This project, carried out in collaboration with centres in some 40 countries, has resulted in a set of documents and materials likely to be useful an usable in a variety of cultural settings, at different levels of health care and by different types of health workers.

There materials include (1) mutually compatible versions of the classification of mental disorders for use at primary health care level, in research, and in clinical psychiatric work with guidelines which facilitate their use; (2) instruments which allow a systematic and unbiased assessment of the mental state, level of disability, history of the disease and other factors of relevance for diagnosis and management of mental disorders; (3) training materials related to the use of ICD and the standardized instruments.

The paper will present the materials produced and give a perspective of future work in this field.
ICD-10, DIAGNOSTIC CATEGORIES, DEVELOPMENT AND USE John Cooper

Meadow Cottage, 25 Ireton Grove, Attenborough, Nottingham, UK

The structure and outline content of Chapter V of ICD-10 will be described, with reference to the main differences between ICD-8, ICD-9, and ICD-10. Comments will also be made on the relationships between ICD-10 and DSM-IV. Unlike ICD-8 and 9, ICD-10 does not use the distinction between neurosis and psychosis as a major principle of classification, and its categories are grouped in 10 main sections that share common descriptive themes. The widespread international process of consultation that was necessary for the production of ICD-10 will also be described briefly.

Different versions of Chapter V have been produced for different purposes. 
THE CLASSIFICATION OF ORGANIC MENTAL DISORDERS ACCORDING TO THE ICD-10

\section{G Mavreas}

University Research Institute of Mental health, University of Athens

Medical School, 74 Vas. Sophias Av., 11528 Athens, Greece

The section F0 of Chapter $V$ of the Tenth Revision of the International Classification of Diseases, dealt specifically with mental disorders the etiology of which van be demonstrated to be due to cerebral disease, brain injury or other insult leading to primary or secondary cerebral dysfunction, with the exception of disorders caused by alcohol or drugs.

The section can be divided into two sub-sections. In the first (F00-F05), there are disorders in which the most prominent features are disturbances of the cognitive functions such as memory, intellect and learning or disturbances of the sensorium such as disturbances of the consciousness and attention. In this group belong the dementias of different origins, amnesic syndromes and delirium. In the second sub-section (F06-F07), the most prominent features are symptoms in the areas of perception, thought, mood or emotion, or in the areas of personality and behaviour. These conditions are phenomenologically similar to those of other sections of chapter V (F2, F3, F4, F6), but a specific cause of organic origin can be demonstrated for all of them.

Specific diagnostic criteria for research have been set for these disorder, enabling their accurate and reliable identification.

ICD-10 TRAINING COURSE

"F10-F19 MENTAL AND BEHAVIOURAL DISORDERS DUE TO PSYCHOACTIVE SUBSTANCE USE"

\section{C.B. Pull}

Centre Hospitalier de Luxembourg, 4 rue E. Barblé, L-1210 Luxembourg, Luxembourg

In ICD-10, block F1 contains a wide variety of disorders that differ in severity (from uncomplicated intoxication and harmful use to obvious psychotic disorders and dementia), but that are all attributable to the use of one or more psychoactive substances (which may or may not have been medically prescribed)

The substance involved is indicated by means of the second and third characters (i.e. the first two digits after the letter F), and the fourth and fifth characters specify the clinical states. To save space, all the psychoactive substances are listed first, followed by the four-character codes; these should be used, as required, for each substance specified but it should be noted that not all four-character codes are applicable to all substances.

The general principles of the classification and the diagnostic guidelines for specifying the clinical stated that are attributable to the use of psychoactive substances are described in the " Clinical descriptions and diagnostic quidelines" or "the blue book". Specific diagnostic criteria for research or DCR for the different clinical states are included in the "Diagnostic criteria for research" or "the green book".

For a standardized assessment of the clinical stated pertaining to flock F1, WHO proposed different global structured diagnostic instruments, including in particular the CIDI and the SCAN, as well as specific instruments such as the CIDI-SAM and the AUDADIS.

Section Fl of the ICD-10 blue book and green book, as well as the instruments connected with section F1, will be presented and discussed.
THE ICD-10 PHC: A NEW CLASSIFICATION FOR MENTAL DISORDERS WITH MANAGEMENT GUIDELINES FOR USE IN PRIMARY CARE

T. Bedirhan Üstün, MD, PD, on behalf of participating centers Scientist, Division of Mental Health, World Health Organization, Geneva, Switzerland

OBJECTIVE: This report gives the initial results of the field trials of the Primary Care Version of International Classification of Diseases (10th Revision) Chapter V for Mental and Behavioural Disorders (ICD-10 PHC)

METHODS: ICD-10 PHC focuses on 24 conditions which are frequently seen in primary care and which can be managed effectively by general practitioners. The classification is accompanied by flipcards one for each of the conditions. The cards contain diagnostic guidelines on one side and management guidelines on the other. The draft ICD-10 PHC was tested in the field for its applicability and acceptance of its style and content. 154 centres from 58 countries participated in the field trials in three different parts: (i) the applicability and usefulness, (ii) reliability and validity, (iii) appropriateness for primary care practice.

SUMMARY OF RESULTS: ICD-10 PHC version proves to be applicable and useful.

CONCLUSIONS: ICD-10 PHC has been developed and finalized in the light of experience of primary care physicians. It will be further tested to show the effectiveness of the classification in terms of training and changing the outcome of mental disorders in Primary Care.

\section{ICD-10 MULTIAXIAL CLASSIFICATION}

\section{A. Janca}

Division of Mental Health, World Health Organization, 1211 Geneva 27, Switzerland

The ICD-10 multiaxial system consists of Axis I, which covers all mental, behavioural and physical disorders included in ICD-10; Axis II, which covers various levels of disability associated with the disorders and is based on the International Classification of impairments, Disabilities and Handicaps; Axis III, which deals with psychosocial, circumstantial and environmental factors influencing the onset, presentation or course of the disorders. it is based on ICD-10 Chapter XXI: Factors influencing status and contact with health services (Z00-Z99).

The ICD-10 multiaxial system has been field tested in more than 70 centres from 26 countries. Clinicians from different parts of the world found the ICD-10 multiaxial system to be applicable, reliable and useful for (a) support of decisions regarding routine care of the patients, (b) training of mental health professionals, (c) research on etiology, course and treatment of mental disorders, and (d) statistical reporting. 


\section{F2 - SCHIZOPHRENIA, SCHIZOTYPAL AND DELUSIONAL DIS- ORDERS \\ John Cooper \\ Meadow Cottage, 25 Ireton Grove, Attenborough, Nottingham, UK}

The structure of this section of Chapter V of ICD-10 will be discussed, and the definitions of its main categories will be reviewed. Differences in the definition of schizophrenia in ICD-10 and in DSM-IV will be highlighted, so as to evaluate their significance. Innovations in F2 that will be discussed include F23, Acute and transient psychotic disorders, F20.4, Post-schizophrenic depression, and F21 Schizotypal disorder.

\section{MOOD DISORDERS, F3}

Horst Dilling

Klinik für Psychiatrie der Medizinischen Universität zu Lübeck, Germany

In Section F3 "Affective Disorders" of ICD-10, Chapter V (F) the core concepts of operationalized diagnosis will be outlined. The differentiation between neurosis and psychosis resp. neurotic and endogenous depression as a fundamental organizing principle has been abandoned. All affective disorders are grouped together and subclassified following course- and severity-related features. Therefore, in this section is differentiated between single episodes, recurrent episodes and persistent disorders, and for the episodes degrees of severity as mild, moderate and severe. Furthermore, a somatic syndrome and psychotic features are introduced describing the severity of the disorders.

During the lecture these classificatory innovations are described comparing the diagnostic approaches of ICD-10 with the traditional concept of ICD-9. Some results of the international field trials introducing the Clinical Descriptions and Diagnostic Guidelines resp. the Diagnostic Criteria for Research are discussed. In this context the results concerning the goodness of fit of the new diagnostic categories and the interrater-reliability coefficients showed an adequate acceptance of the classificatory changes.
THE ICD-10 FIELD TRIALS IN EUROPE

T. Bedirhan Üstün, MD, PD on behalf of participating centers, Scientist, Division of Mental Health, World Health Organization, Geneva, Switzerland

OBJECTIVE: This report summarizes results of the field trials Research version of the International Classification of Diseases (10th Revision) Chapter V for Mental and Behavioural Disorders (ICD-10 DCR) in Europe METHODS: In preparing for the Tenth Revision of the international Classification of Diseases (ICD-10), the Division of Mental Health of the World Health Organization developed several versions of chapter $V$, dealing with mental and behavioural disorders. The version for research purposes is called the "Diagnostic Criteria for Research" (ICD-10 DCR) and gives operational criteria for diagnosis of categories included in ICD-10 Chapter V. An international field trial was undertaken by the WHO's Division of Mental Health to evaluate draft criteria and refine them further. Data were obtained to assess the inter-rater reliability, the confidence with which diagnoses could be made and the ease of use of the criteria. Additional sub-studies checked the concordance between the DCR and ICD-10 Clinical Descriptions and Diagnostic Guidelines and other national classification systems. The field trial was carried out at 151 clinical centres in 33 countries, by 942 clinicians who conducted 11,491 individual assessments.

SUMMARY OF RESULTS: ICD-10 DCR version proves to be useful and very practical for research purposes. Most researchers reported that the draft diagnostic criteria were explicit and easy to apply and inter-rater reliability was high for most categories. For some categories such as those dealing with some polymorphic psychotic disorders or milder forms of affective disorders, criteria were rated as somewhat difficult to use, and reliability was lower. Comparison of results of the DCR-10 field trials with those of the Clinical Descriptions and Diagnostic Guidelines demonstrated that for some diagnoses there are substantial increases in inter-rater reliability when the DCR-10 is used.

CONCLUSIONS: ICD-10 DCR has been developed and finalized in the light of experience of the application of diagnostic criteria to real clinical cascs. The higher levels of agreement are probably explained by the provision of precise definitions which increases the agreement between assessors.

\section{F4 NEUROTIC, STRESS RELATED AND SOMATOFORM DISORDERS AND F5 BEHAVIOURAL SYNDROMES ASSOCIATED WITH PHYSIOLOGICAL DISTURBANCES AND PHYSICAL FACTORS \\ JJ Lopez-Ibor, Jr.}

Psychiatric Department. Complutense University. Martín Lagos s/n. 28035, Madrid, Spain

The classification of ICD-10 categories F4 (Neurotic, stress related and somatoform disorders) and F5 (Behavioural syndromes associated with physiological disturbances and physical factors) is always difficult and ICD-10 has solved this challenge with remarkable efficacy. The difficulties are due to the following aspects: 1) In the past pathogenic theories were used to define these disorders even around an almost unique feature such as anxiety; 2) The difficulty of differentiating these disorders from ordinary life conditions; 3) The handicap associated to the disorder. In the post DSM-III era the symptoms and clinical features present at the bases of psychiatric diagnosis and this approach has been fruitful even in categories such as those mentioned. The results of field trials with ICD-10 draft version showed a high inter-rater reliability for these conditions: The kappa index for F4 was .74 and for F5 .91 (the global kappa for ICD-10 two character categories was .81). There are several differences between ICD-10 and DSM-IV, some of them very important as the relationship between panic attacks and agoraphobia and the presence of neurasthenia in ICD-10. 
ICD-10 AND MENTAL RETARDATION

MG Gelder

Professor and Head of Department, Oxford University Department of Psychiatry, Warneford Hospital, Oxford OX3 7JX

Chapter V of ICD-10 dealt with mental retardation in a brief and simple way: only degree of retardation and impairment of behaviour can be coded under F7. For the full diagnosis of a mentally retarded person it is usually necessary to record also (a) concurrent mental disorder, (b) disorder of psychological development, (c) associated physical disorder and (d) abnormal psychosocial factors. For some of these additional diagnoses the criteria in ICD-10, formulated for patients of normal intelligence, are difficult to apply to mentally retarded patients. This paper will describe the development of a multi-axial system designed specifically for use in clinical practice with mentally handicapped patients. The multi-axial system will be reviewed and examples given of the way in which diagnostic criteria and practices have been modified to meet the needs of those working with mentally retarded people
SCAN: SCHEDULES FOR CLINICAL ASSESSMENT IN NEUROPSYCHIATRY

J. K. Wing

Royal College of Psychiatrists Research Unit, 11 Grosvenor Crescent, London SW1X 8PG, UK

Scan represents the latest stage in a 30-year line of development that began in the late 1950s. The ninth edition of the Fresent State Examination (PSE9) was the first to be published for general use, following 15 years of work on earlier versions, including two large multicentre international projects the US-UK Diagnostic Project and the International Pilot Study of Schizophrenia. It was also the first to have computerised algorithms for deriving an ICD-9 diagnosis. It has been widely used, as evidenced by its translation into 35 or more languages. Reliability under a wide variety of conditions is high.

SCAN is a set of instruments aimed at assessing, measuring and classifying the psychopathology and behaviour associated with the major psychiatric disorders of adult life. It has 4 components: the tenth edition of the Present State Examination (PSE-10), the Glossary of Differential Definitions, the Item Group Checklist (IGC) and the Clinical History Schedule (CHS). Pse10 itself has two parts. Part I covers somatoform, dissociative, anxiety, depressive and bipolar disorders, and problems associated with appetite, alcohol and other substance use. There is a screen for Part II conditions. Part II covers psychotic and cognitive disorders and observed abnormalities of speech, affect and behaviour.

The computer programme provides scores and symptom profiles, and diagnoses for ICD-9, ICD-10, DSM-III-R and DSM-IV.

There are Training Centres accredited by WHO in various parts of the world. In Europe, the centres are in Aarhus, Athens, Cardiff, Groningen, Leicester, London, Lübeck, Luxembourg, Mannheim, Nottingham and Santander.

\section{ICD-10 DISORDERS OF PERSONALITY AND BEHAVIOUR, F6} Aly A. Dahl

Gaustad Hospital, Institute Group of Psychiatry, University of Oslo, Norway

The changes from ICD-9 to ICD-10 within the group of personality disorders are described. The attempts at co-ordination with DSM-IV personality disorders are discussed. The separate personality disorders are presented. A self-report screening instrument as well as a structured interview for the diagnoses of ICD-10 personality disorders are introduced.
THE CIDI AS ASSESSMENT INSTRUMENT FOR ICD-10 DIAGNOSTICS

HU Wittchen

Max Planck Institute of Psychiatry, Clinical Institute, Kraepelinstr. 2. 10, D-80804 München, Germany

The CIDI is a comprehensive and fully standardized diagnostic interview designed for assessing mental disorders according to the definitions of the Diagnostic Criteria for Research of ICD-10 and DSM-III-R. The instrument contains 276 symptom questions many of which are coupled with probe questions to evaluate symptom severity, as will as questions as for assessing help-seeking behaviour, psychosocial impairments, and other episode-related questions. Although primarily intended for use in epidemiological studies of mental disorders, it is also being used extensively for clinical and other research purposes. The review documents the wide spread use of the instrument and discusses several test-retest and interrater reliability studies of the CIDI. Both types of studies have confirmed good to excellent Kappa coefficients for most diagnostic sections. In international multicenter studies as well as several smaller center studies the CIDI was judged to be acceptable for most subjects and was found to be appropriate for use in different kinds of settings and countries. There is, however, still a need for reliability studies in general population samples, the area the CIDI was primary intended for. Only a few selected aspects of validity have been examined so far, mostly in smaller selected clinical samples. The need for further procedural validity studies of the CIDI with clinical instruments such as the SCAN as well as cognitive validation studies is emphasized. The latter should focus on specific aspects, such as the use of standardized questions in the elderly, cognitive probes to improve recall of episodes and their timing, as well as the role of order effects in the presentation of diagnostic sections. 
ICD-10 CHILD AND ADOLESCENT PSYCHIATRY

Eric Fombonne

Senior Lecturer and Honorary Consultant

Institute of Psychiatry, Department of Child and Adolescent Psychia-

try, De Crespigny Park, London SE5 8AF, England

In this lecture, the section F70-79 (mental retardation), F80-89 (developmental disorders) and F90-99 (emotional and behavioural disorders with an onset in childhood or adolescence) will be presented, both for their clinical version and research version of ICD-10. Emphasis will be placed on the change that occur in ICD-10 as compared to the prior available nosographies such as ICD-9 and DSM-III. Thus as compared to ICD-9, the format of the glossary available in ICD-10 has been expanded and each category of disorder starts with a discussion of the main conceptual issues involved in the specification of this class of disorders, with an account of the main difficulties in differential diagnosis. Each disorder within a given category is now described with detailed symptom descriptions and provides the user with guidelines and steps to be followed to reach a particular diagnosis. With the success of using specific diagnostic criteria, there is now a higher level of specification of the symptoms needed to reach a diagnosis. As compared to ICD-9, some categories have been revised and new categories have been added. Examples of these changes will be given. Collaborative work has been undertaken between members of the ICD-10 and DSM-IV task force in order to bring these two classifications to a closer agreement. Thus we shall illustrate areas where DSM-IV and ICD-10 will be closely comparable and others where discrepancies will remain. Examples where discrepancies will persist across the two classifications are for comorbid disorders, such as conduct disorders which frequently occur in conjunction with either depression or hyperkinesis. Finally, the interest of ICD-10 for teaching purposes will be outlined. 